\title{
Six new species of Agrilus Curtis, 1825 (Coleoptera, Buprestidae, Agrilinae) from the Oriental Region related to the emerald ash borer, A. planipennis Fairmaire, 1888 and synonymy of Sarawakita Obenberger, 1924
}

\author{
Eduard Jendek ${ }^{1, \dagger}$, Maria Lourdes Chamorro ${ }^{2, \ddagger}$ \\ I Ottawa Plant Laboratory, Canadian Food Inspection Agency, K.W. Neatby Bldg., 960 Carling Avenue, \\ Ottawa, Ontario, K1A 0C6, Canada 2 Systematic Entomology Laboratory, United States Department of Agri- \\ culture, National Museum of Natural History, 10th \& Constitution Ave NW, Washington, DC 20560, USA \\ † urn:lsid:zoobank.org:author:963E83FF-C75C-41DC-AC42-22854A1D6C9A \\ ¥ urn:lsid:zoobank.org:author:8BD38884-6514-4D9E-BDFB-72A41DB11C2C \\ Corresponding author: Maria Lourdes Chamorro (lourdes.chamorro@ars.usda.gov)
}

Academic editor: T. Erwin | Received 6 September 2012 | Accepted 2 November 2012 | Published 8 November 2012

urn:lsid:zoobank.org:pub:AFA2A649-82D9-4981-883E-480D3E7901DE

Citation: Jendek E, Chamorro ML (2012) Six new species of Agrilus Curtis, 1825 (Coleoptera, Buprestidae, Agrilinae) from the Oriental Region related to the emerald ash borer, A. planipennis Fairmaire, 1888 and synonymy of Sarawakita Obenberger, 1924. ZooKeys 239: 71-94. doi: 10.3897/zookeys.239.3966

\begin{abstract}
Six new species of Agrilus Curtis, 1825 with affinities to the emerald ash borer, A. planipennis Fairmaire, 1888, are described from the Oriental Region: $A$. crepuscularis sp. n. (Malaysia); $A$. pseudolubopetri sp. n. (Laos); A. sapphirinus sp. n. (Laos); A. seramensis sp. n. (Indonesia); A. spineus sp. n. (Malaysia); and $A$. tomentipennis sp. n. (Laos). The genus Sarawakita Obenberger, 1924 syn. nov. is considered a junior synonym of Agrilus.
\end{abstract}

\section{Keywords}

Agrilus, Buprestidae, emerald ash borer, new species, synonym, taxonomy, nomenclature, Asia

Copyright Eduard Jendek, Maria L. Chamorro. This is an open access article distributed under the terms of the Creative Commons Attribution License 3.0 (CC-BY), which permits unrestricted use, distribution, and reproduction in any medium, provided the original author and source are credited. 


\section{Introduction}

The current study stems from an international, multi-agency effort between the following institutions (listed in alphabetical order): the Canadian Food Inspection Agency, the Chinese Academy of Sciences Institute of Zoology, the United States Department of Agriculture, and the Zoological Institute, Russian Academy of Sciences to understand the evolutionary relationships and biology of the highly invasive Agrilus planipennis Fairmaire, 1888 (emerald ash borer - EAB) and its relatives. This effort aims to determine, define, and illustrate the characters that enable identification of EAB and a core group of related species; make predictions about potential new invasive species with similar evolutionary histories and adaptations; educate the public and other scientists; and contribute knowledge needed to develop control strategies to manage outbreaks. A comprehensive, illustrated identification manual presenting these findings is underway. In this paper we describe six new species related to $A$. planipennis and propose new taxonomic and nomenclatural acts discovered during the course of our study.

\section{Materials and methods}

Terminology, morphology, format and style of descriptions follow Jendek and Grebennikov (2011). Square brackets "[ ]" are used for our remarks and addenda. The following equipment was used for observation and imaging: Leica (Wetzlar, Germany) MZ Apo stereomicroscope and Zeiss (Oberkochen, Germany) Discovery v20 stereomicroscope with AxioCam HRc, respectively.

Abbreviations for collections

$\begin{array}{ll}\text { EJCB } & \text { Jendek, E., Bratislava, Slovak Republic [presently in Ottawa, Canada] } \\ \text { MNHN } & \begin{array}{l}\text { Muséum National d'Histoire Naturelle, Paris, France (Bruneau de Miré, } \\ \text { I; Mantilleri, A.) }\end{array} \\ \text { NMPC } & \begin{array}{l}\text { Národní Museum (Natural History), Prague, Czech Republic (Kubáň, V.) } \\ \text { USNM }\end{array} \\ & \begin{array}{l}\text { National Museum of Natural History, Washington D.C., USA (Lin- } \\ \text { gafelter, S.W.) }\end{array}\end{array}$

\section{Taxonomic section}

\section{Agrilus Curtis, I 825}

= Sarawakita Obenberger, 1924 syn. $\mathbf{n}$.

Obenberger 1924: 39-40, figs 15, 41 (proposed as genus; Type species: Sarawakita latifrons Obenberger, 1924 fixed by original designation and monotypy) - Obenberger 1936: 1085 (world catalog) - Kubáň et al. 2000: 196 (valid genus; Agrilini) - Bellamy 2003: 2380 (valid genus; Agrilini incertae sedis) 
Agrilus hewitti Kerremans, 1912, comb. rest.

http://species-id.net/wiki/Agrilus_hewitti

Figs $1-8$

Kerremans, 1912: 74 (Agrilus, description) - Obenberger 1936: 1085 (world catalog) - Obenberger 1960: 125-126 (type examination; redescription) - Jendek 2006: 34 (Sarawakita; lectotype designation; synonymy) - Bellamy 2008: 2380 (Sarawakita; world catalog).

= latifrons Obenberger, 1924

Obenberger 1924: 40 (Sarawakita, description) - Jendek 2006: 34 (Sarawakita; synonym of hewitti; lectotype designation) - Bellamy 2008: 2380 (Sarawakita; synonym of hewitti).

Material examined. Type material. See Jendek (2006).

Other material. 1 (EJCB): "Sarawak 1897"; 1 (EJCB): "Malaysia, Pahang, 2000, Cameron Highlands, Tanah Rata, 1600m, J. Horák leg. 26.1.-10.2.”

\section{Agrilus daillieri Baudon, 1965}

http://species-id.net/wiki/Agrilus_daillieri

Figs 9-11

Baudon, 1965: 223-224 (Agrilus, description) - Baudon 1963: 54 ([Note: Unavailable name, cited without characters]) - Descarpentries and Villiers 1967: 149 (sinensis species group) - Baudon 1968: 135, 168 (characters in key; Laos) - Ohmomo 2002: 23 (faunal records; Thailand) - Bellamy 2008: 2057 (world catalog).

Material examined. Type material. Holotype $\widehat{\partial}$, (MHNB): "Pak Ca Dinh 15.v.[19]63 [h] Laos (Baudon) [p] \Type [p] [red label] \Agrilus daillieri mihi Type [h] A. Baudon det. [p] [blue label]".

Other material. 1 (ZIN): "Vietnam, Vinh-Phu Prov. Tam-Dao V-VI.1997, N. L. Orlov leg."; 1 (EJCB): "N Vietnam (Tonkin) pr. Hoang Lien Son, SA PA 11-15.v.1990, Vit Kubáň leg."; 1 (EJCB): "Vietnam, Tam Dao, Vinh-Phu Pr., 3-11.6.1985, Navrátil lgt., Collectio Vit Kubáń"; 1 (EJCB): "Vietnam, 1100-1700, 22.18N, 103.50E, W SaPa, 29.V-11.VI.1996, lg. K.W. Anton"; 1 (EJCB): "N. Vietnam, $21^{\circ} 27 \mathrm{~N}, 105^{\circ} 39 \mathrm{E}, 70 \mathrm{~km} \mathrm{NW}$ of Hanoi, Tam Dao, 9-19.v.1996, 900-1200m, Dembicky \& Pacholátko leg."; 1 (EJCB): "North Vietnam, Tam Dao, 28.vii.1997".

Remarks. Upon examination of the material mentioned above and the type specimens of Agrilus hewitti, Sarawakita latifrons and Agrilus daillieri we have come to the conclusion that Sarawakita should be treated as a junior synonym of Agrilus.

Agrilus daillieri and $A$. hewitti are very closely related, large $(>10 \mathrm{~mm})$ and robust species which share many morphological features with $A$. planipennis. Their taxonomic position will be analyzed in detail in the upcoming revision. 


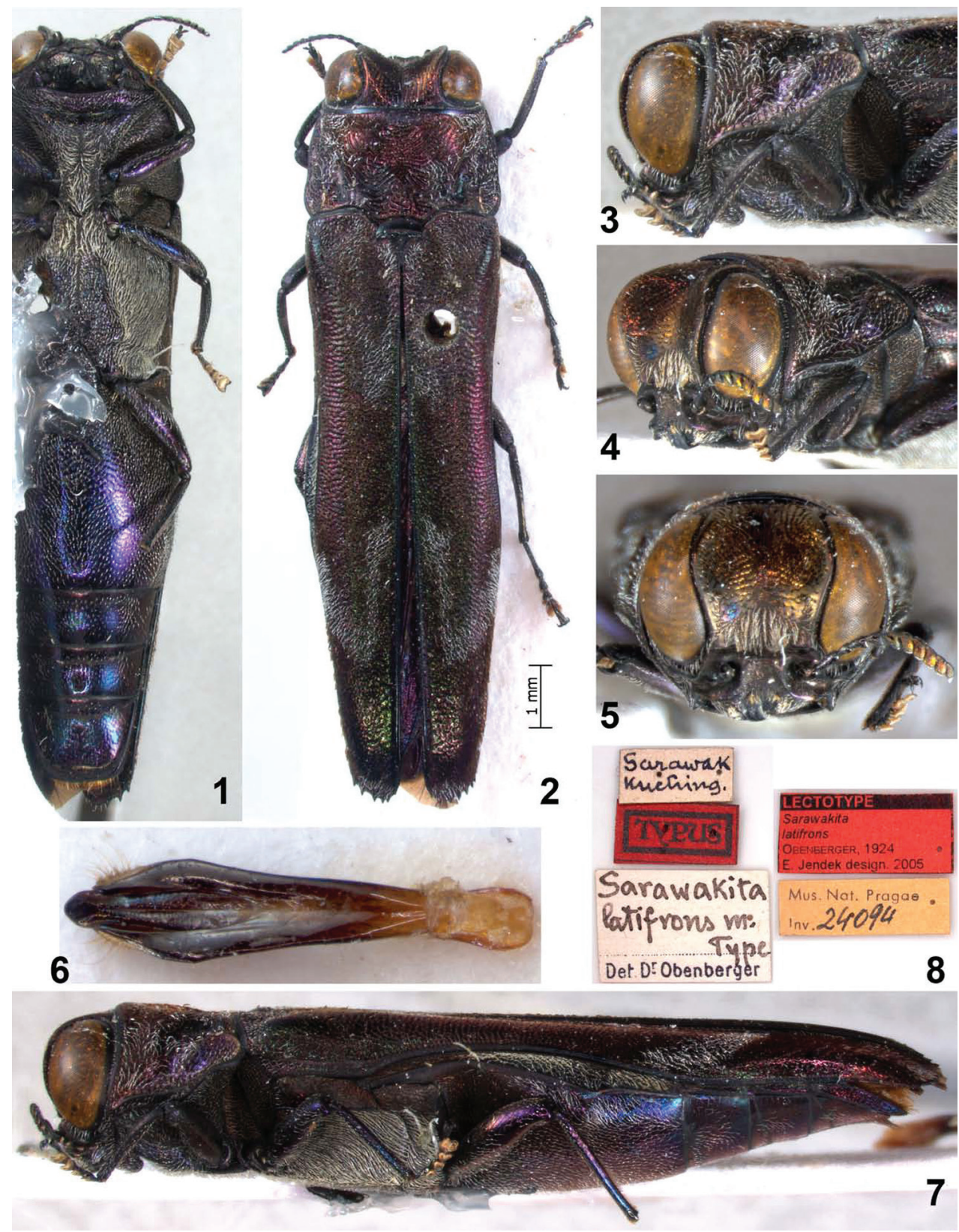

Figures I-8. Agrilus hewitti Kerremans. Holotype: I ventral view $\mathbf{2}$ dorsal view $\mathbf{3}$ lateral view of head and pronotum $\mathbf{4}$ oblique-lateral view of head and pronotum $\mathbf{5}$ anterior view of head $\mathbf{6}$ dorsal view of aedeagus $\mathbf{7}$ lateral view $\mathbf{8}$ type labels. 


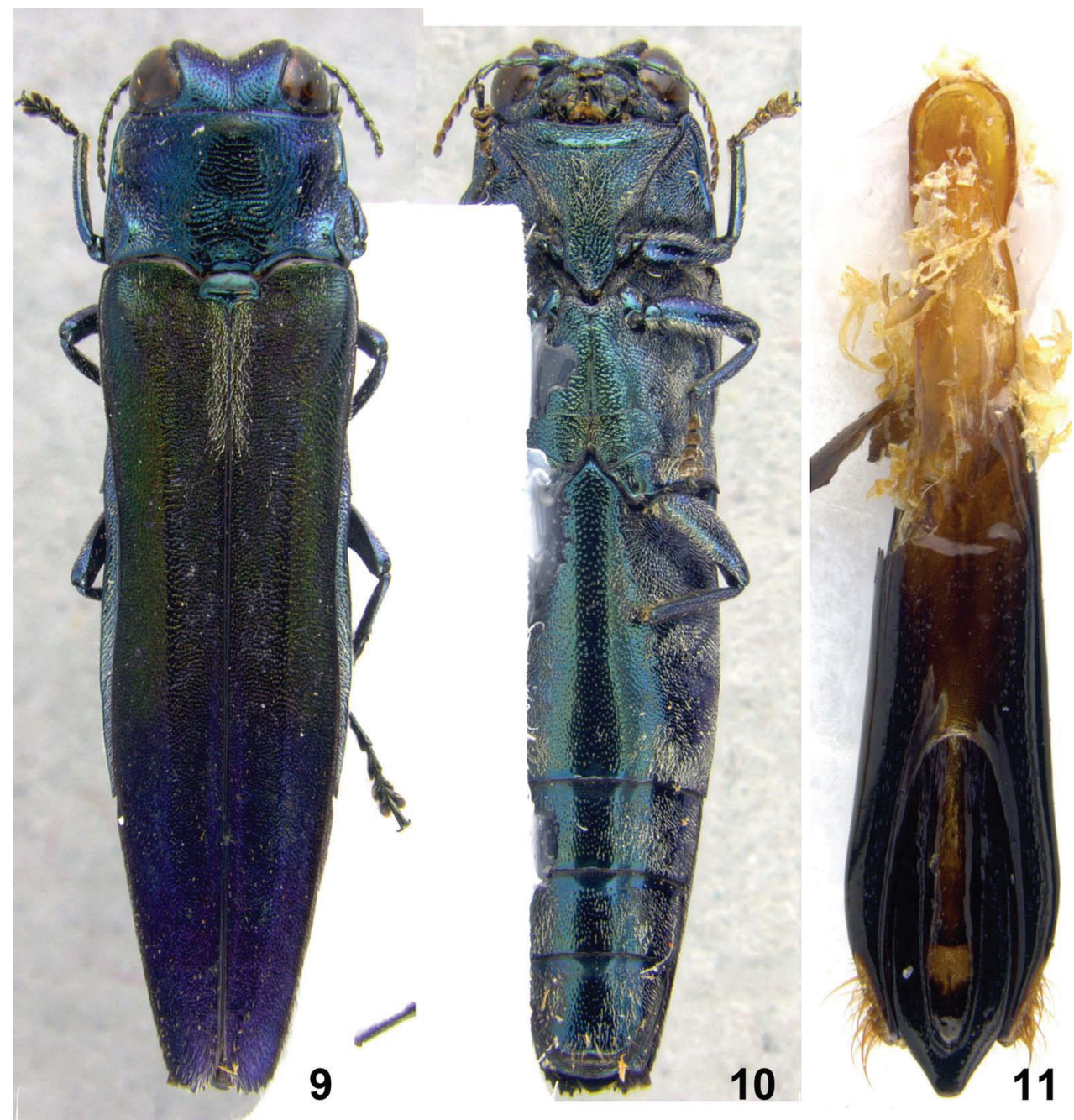

Figures 9-I I. Agrilus daillieri Baudon. Holotype: 9 dorsal view 10 ventral view I I dorsal view aedeagus.

\section{Agrilus crepuscularis Jendek \& Chamorro, sp. n.}

urn:lsid:zoobank.org:act:E04AB2A3-B27B-4B31-A6A4-123E124B7292

http://species-id.net/wiki/Agrilus_crepuscularis

Figs 12-23

Diagnosis. This species resembles $A$. planipennis by the body shape; transverse and trapezoid pronotum; obvious medial pronotal impression; very narrow marginal and submarginal interspace; rounded elytral apices; and by the small scutellum. Agrilus crepuscularis sp. n. can be distinguished from $A$. planipennis mainly by the missing prehumerus; presence of obvious, yellow pubescence ventrally and by the rounded not spined apex of pygidium.

Description. BODY: Size: $10 \mathrm{~mm}$ (Holotype); Shape: cuneiform; Build: slender. 

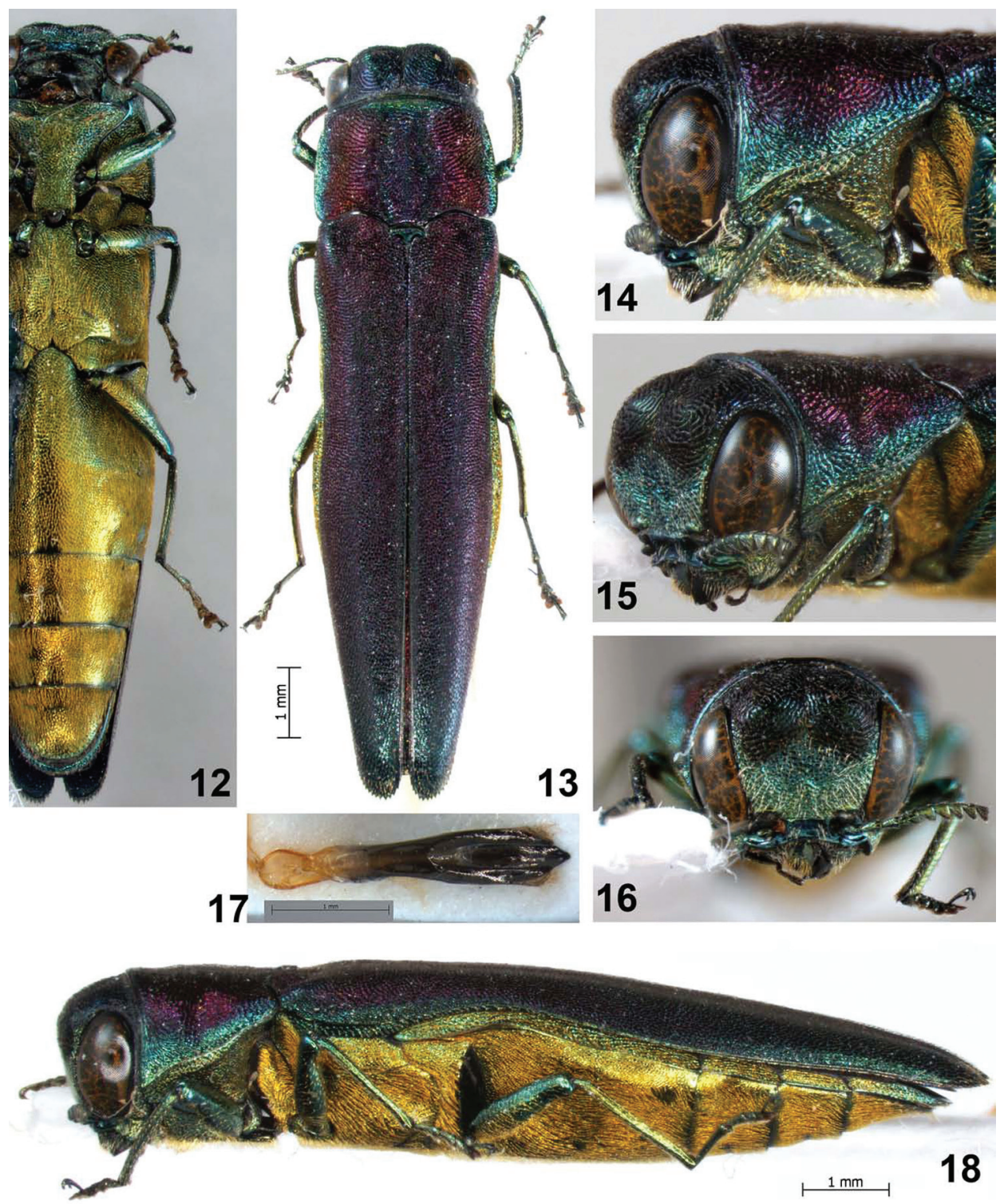

Figures I 2-18. Agrilus crepuscularis Jendek \& Chamorro, sp. n. Holotype male: $\mathbf{2}$ ventral view $\mathbf{3}$ dorsal view $\mathbf{4}$ lateral view of head and pronotum $\mathbf{I 5}$ oblique-lateral view of head and pronotum $\mathbf{1 6}$ anterior view of head $\mathbf{I} \mathbf{7}$ dorsal view of aedeagus $\mathbf{I} 8$ lateral view.

HEAD: Shape: obviously flat; Medial impression (depth): deep; Medial impression (extent): vertex; Epistoma: with raised upper margin; Frons: Shape: markedly convex; Outline: protruding from head outline; Vertex: Outline: slightly protruding from head outline; Sculpture: punctures, semispherical, dense, rough; Eyes: Size: large; Shape: protruding from head outline; Lower margin: in line with antennal socket; Antennae: Length: moderate; Shape: slender. 

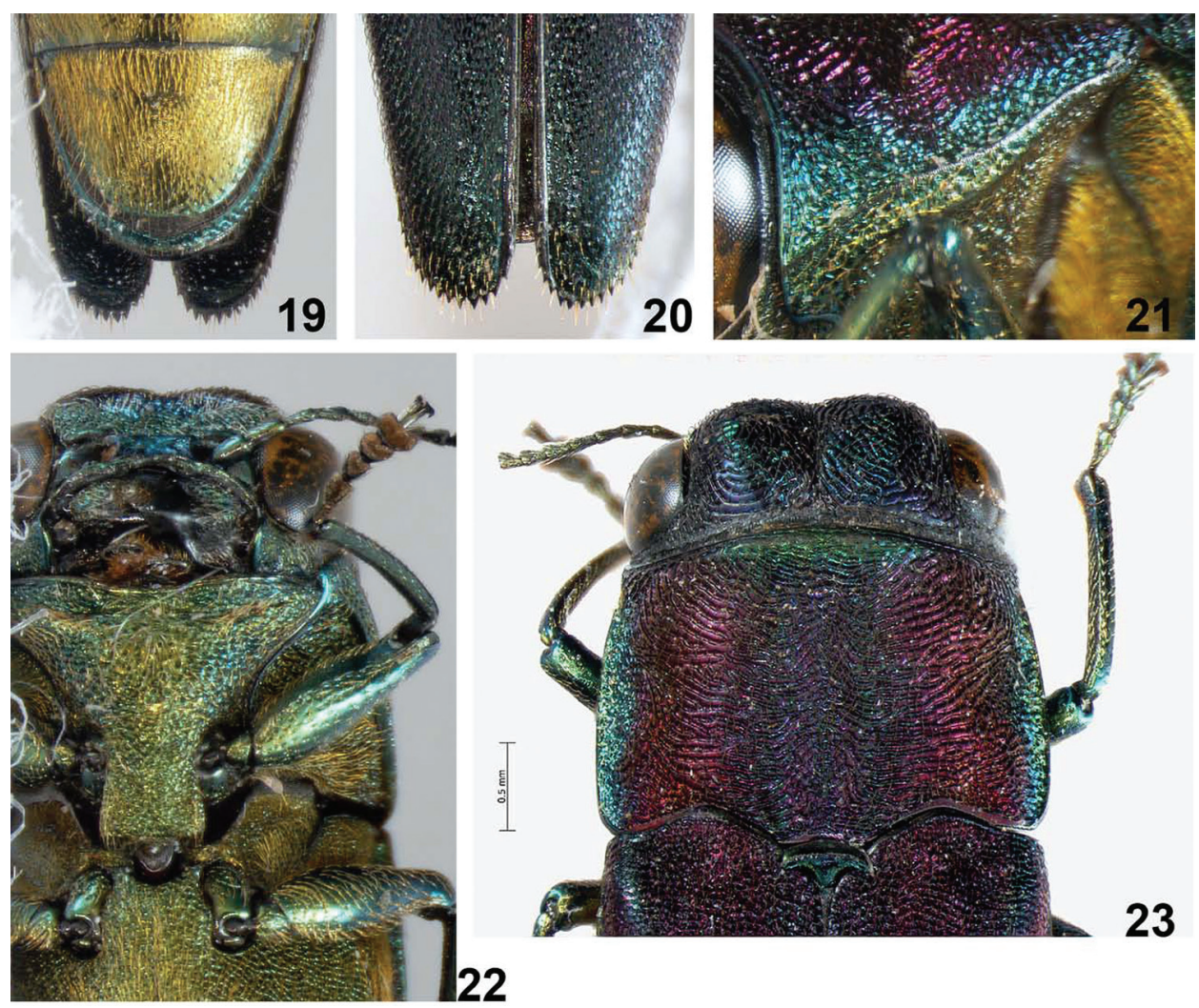

Figures 19-23. Agrilus crepuscularis Jendek \& Chamorro, sp. n. Holotype male: 19 last abdominal ventrite $\mathbf{2 0}$ elytral apices $\mathbf{2} \mathbf{I}$ oblique-lateral view of marginal and submarginal carinae $\mathbf{2 2}$ ventral view of head and prosternum $\mathbf{2 3}$ dorsal view of head and pronotum.

PRONOTUM: Shape: visually square; Sides: markedly arcuate; Maximal width: at middle; Anterior margin: narrower than posterior; Anterior lobe: moderate; Shape: arcuate; Position: at level with anterior pronotal angles; Posterior angles: Apex: blunt, Shape: obtuse; Disk: Convexity: flat, Disk impressions: Presence: medial and lateral, Medial impressions (shape): entire, Medial impressions (depth): deep; Lateral impressions (Depth and width): shallow and broad; Prehumerus: absent; Marginal and submarginal carinae: Interspace: narrow; Convergence: strongly convergent; Junction: present; Scutellum: Size: small, Disk: impressed, Scutellar carina: present.

ELYTRA: Color: unicolored; Humeral carina absent; Apices: Arrangement: separate; Width: wide. Shape: arcuate.

STERNUM: Prosternum: with long yellow pubescence in males; Prosternal lobe: Size: moderately sized; Anterior margin: arcuately emarginate; Emargination wide and moderately deep; Prosternal process: Shape: dilated; Sides: arcuate; Angles: acute; Disk flat; Metasternal projection: flat.

ABDOMEN: Sternal groove: Shape on apex of last ventrite: arcuate; Pygidium: Apical margin: arcuate. 
LEGS: Metatarsus: somewhat longer than mesotarsus; Tarsomere 1: subequal to or longer than 2-4 combined.

GENITALIA: Aedeagus: Symmetry: symmetrical.

Type locality. Malaysia, Pahang state, $35 \mathrm{~km}$ Southwest Kuala Rompin, 2.617N, 103.337E, Endau Rompin State Park.

Type specimens. Holotype, ${ }^{\lambda}$, (EJCB): "Malaysia, Pahang, 28.ii-13.iii, $35 \mathrm{~km}$ SW Kuala Rompin, 2.617N, 103.337E, 50 m, Endau Rompin State Park, E. Jendek leg. 2011”.

Distribution. Malaysia: Pahang state.

Etymology. The specific name is derived from the Latin crepusculum (twilight). It refers to the collecting circumstances with the holotype landing on the sheet when collecting at light.

\section{Agrilus pseudolubopetri Jendek \& Chamorro, sp. n.}

urn:lsid:zoobank.org:act:7E1BC733-2C05-4D96-94FA-0964E91548E2

http://species-id.net/wiki/Agrilus_pseudolubopetri

Figs 24-39

Diagnosis. The male resembles $A$. lubopetri Jendek, 2000 in color, shape and size; however, the following characters distinguish the males of the two species: Agrilus pseudolubopetri sp. n. does not have expanded elytral apices and lacks white pubescence; the interspace between marginal and submarginal pronotal carinae is broader anteriorly in A. pseudolubopetri; and the aedeagus is broader subapically. Female can be distinguished from females of $A$. lubopetri by larger, more robust size, purple color (sometimes green to copper), and by unexpanded elytral apices. The orange pubescence on the pronotal sides of $A$. pseudolubopetri is markedly less extensive than that in A. lubopetri.

Description. BODY: Size: 14-18 mm (Holotype $17 \mathrm{~mm}$ ); Shape: cuneiform; Build: slender.

HEAD: Shape: obviously flat; Medial impression: deep; Extent: frons; Epistoma: with raised upper margin; Frons: Shape: flat; Outline: not protruding from head outline; Vertex: Outline: not protruding from head outline; Sculpture: punctures; Density: sparse; Intensity: superficial; Eyes: Size: large; Shape: not protruding from head outline; lower margin below antennal socket; Antennae: Length: long (males), medium (females), Shape: slender.

PRONOTUM: Shape: transverse; Sides: markedly arcuate; Maximal width: at middle; Anterior margin: wider than posterior; Anterior lobe: vague; Shape: arcuate; Position: at level with anterior pronotal angles; Posterior angles: Apex: sharp, Shape: acute; Disk: Convexity: flat; Disk impressions: Presence: medial and lateral, medial impression (shape): entire; lateral impressions: shallow and wide; Prehumerus: absent; Marginal and submarginal carinae: Interspace: narrow; Convergence: strongly convergent; Junction: present; Scutellum: Size: rudimentary, Disk: not impressed, Scutellar carina: obsolete or present. 

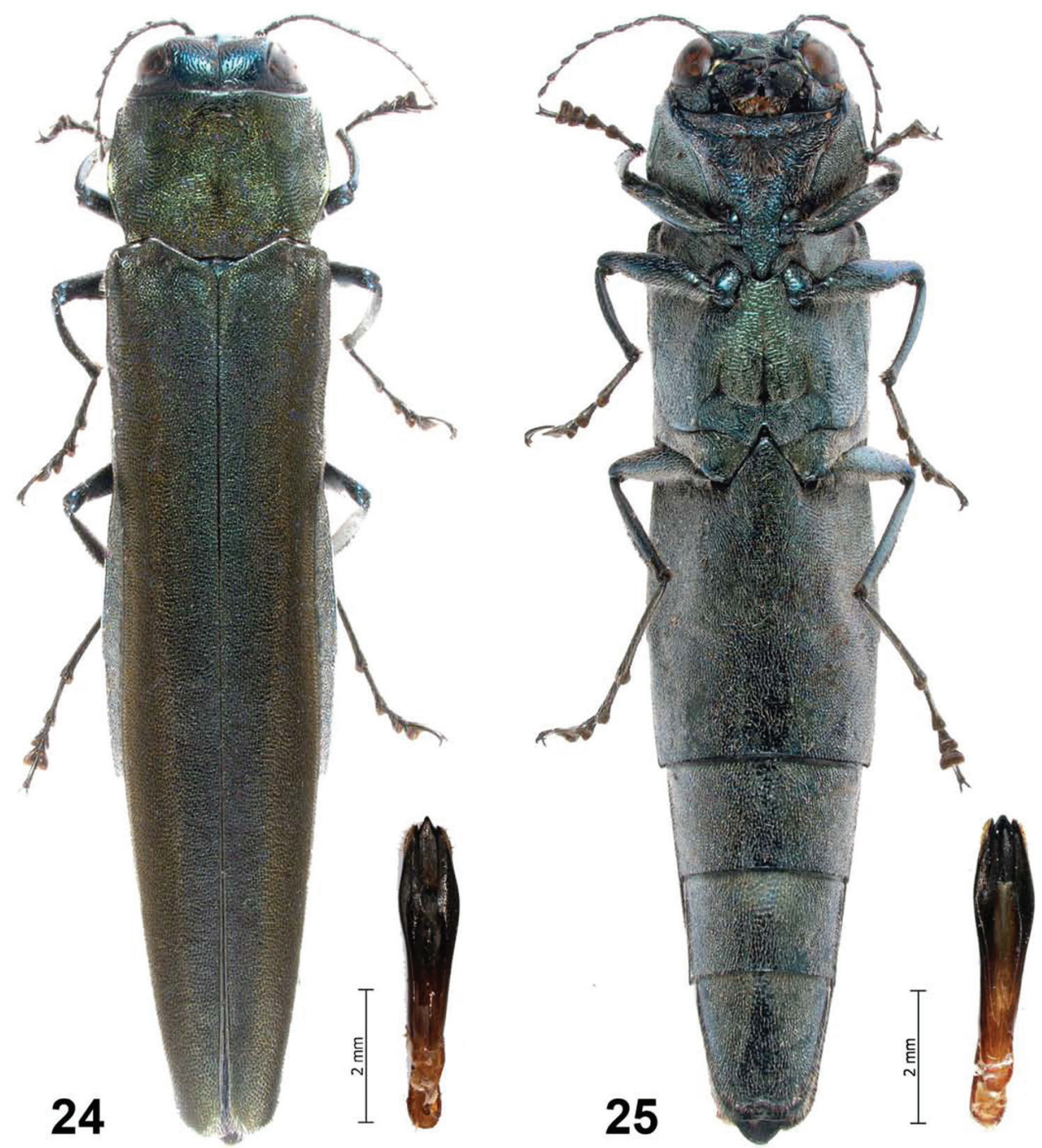

Figures 24-25. Agrilus pseudolubopetri Jendek \& Chamorro, sp. n. Male habitus and aedeagus: 24 dorsal view 25 ventral view.

ELYTRA: Color: unicolored; Humeral carina absent; Apices: Arrangement: separate; Shape: subangulate, Modifications: margin denticulate; Elytral pubescence: distal only; Distal: apical. STERNUM: Sexual modification in male: with longer white pubescence; Prosternal lobe: Size: moderate; Anterior margin: arcuately emarginate; Emargination: Depth: moderately deep; Width: wide; Prosternal process: Size: moderate; Shape: narrowed; Angles: absent; Disk: flat; Metasternal projection: flat.

ABDOMEN: Sternal groove: Shape on apex of last ventrite: arcuate, Depth: shallow; Width: narrow; Pygidium (apical margin): arcuate; Last ventrite (apical margin): subtruncate. 

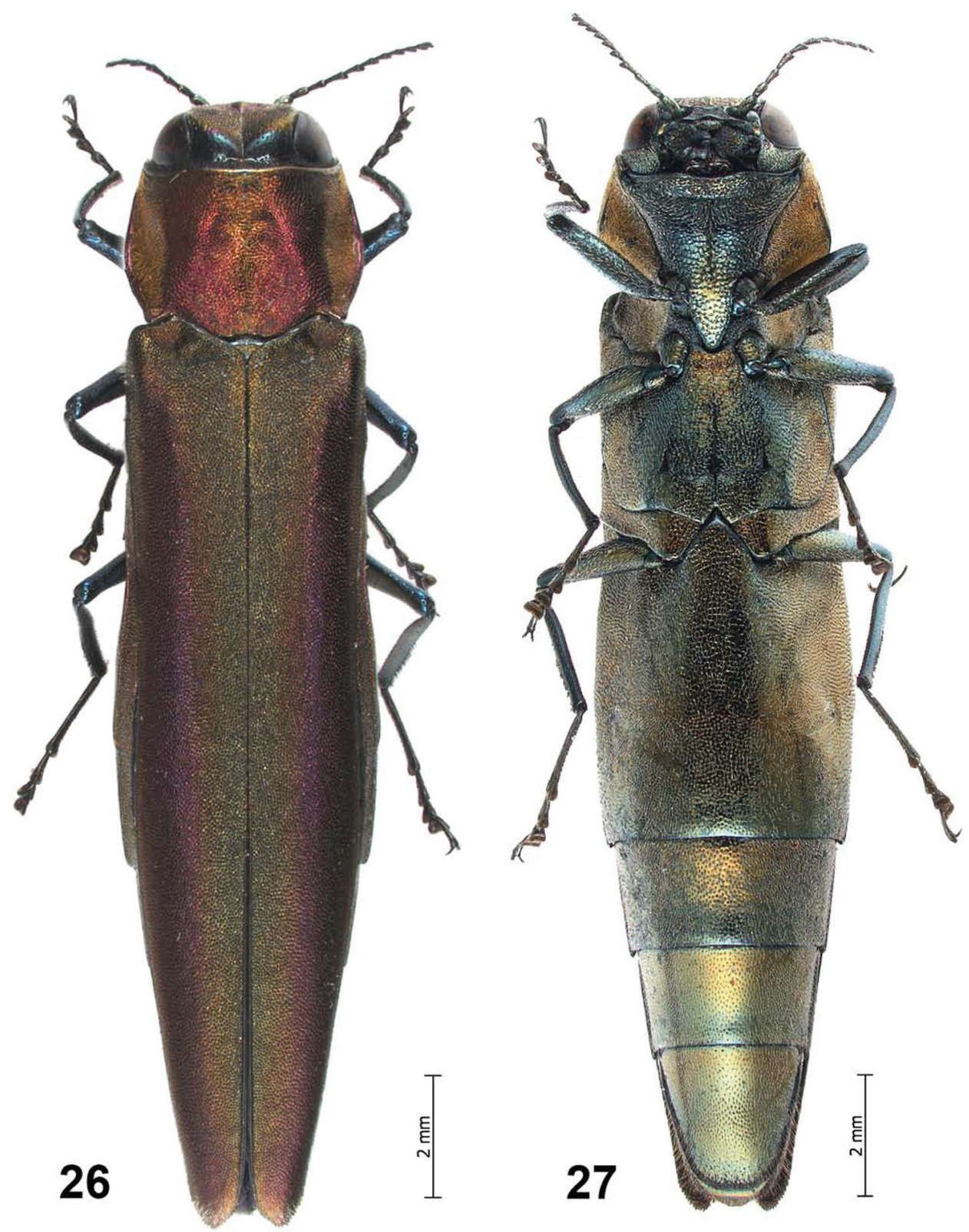

Figures 26-27. Agrilus pseudolubopetri Jendek \& Chamorro, sp. n. Female habitus: 26 dorsal view 27 ventral view.

LEGS: Metatarsus: distinctly longer than mesotarsus; Metatarsomere 1: longer than 2-4 combined.

GENITALIA: Aedeagus: Symmetry: symmetrical; Ovipositor: elongate. 

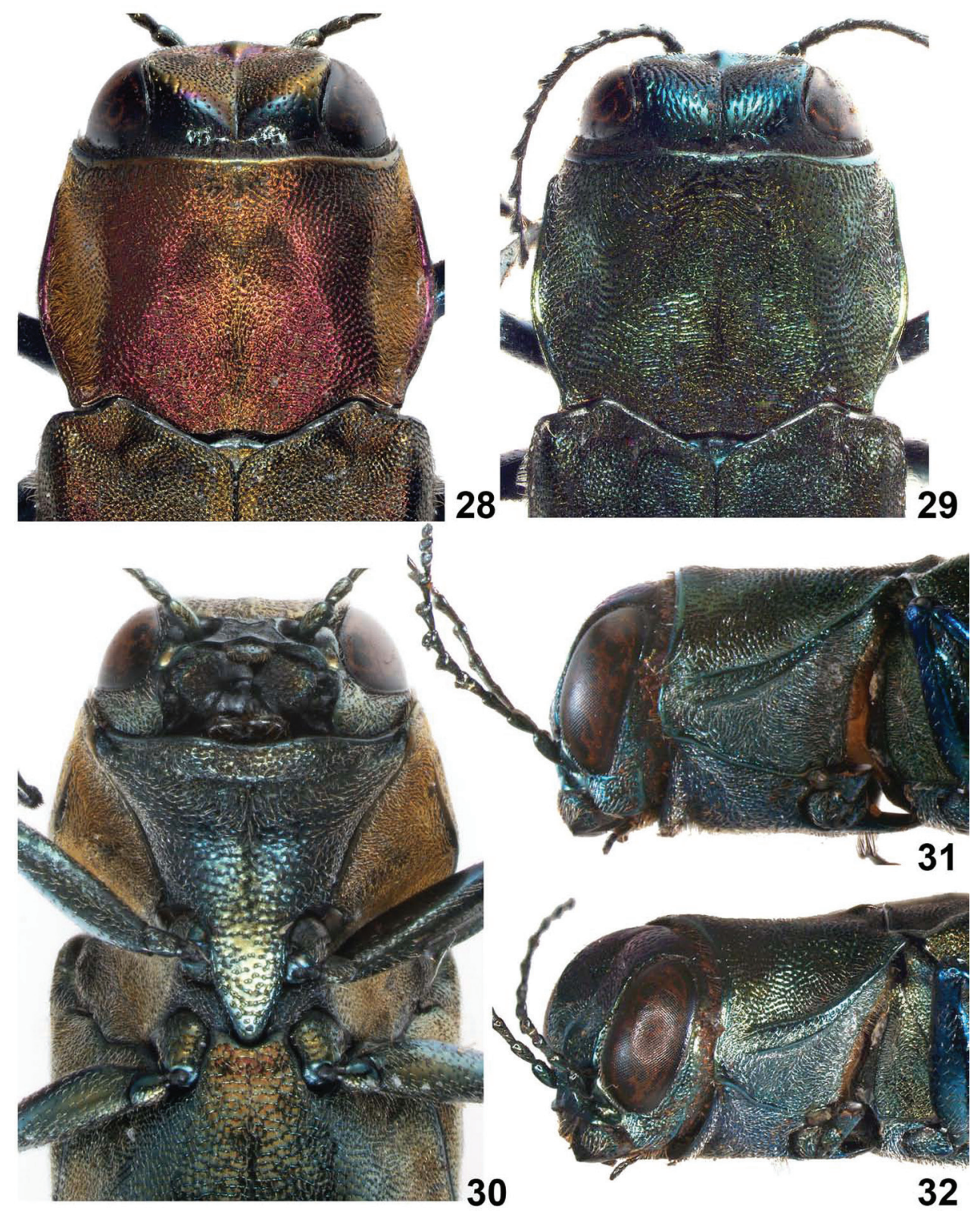

Figures 28-32. Agrilus pseudolubopetri Jendek \& Chamorro, sp. n. 28 female, dorsal view of head, pronotum, scutellum 29 male, dorsal view of head, pronotum, scutellum $\mathbf{3 0}$ female, ventral view of head and sternum $\mathbf{3}$ I male, lateral view of head and pronotum $\mathbf{3 2}$ male, oblique-lateral view of head and pronotum.

Type locality. Northeastern Laos, Hua Phan Province, $\sim 20^{\circ} 12^{\prime} \mathrm{N}, 104^{\circ} 01^{\prime} \mathrm{E}$, Phu Phan Mt.

Type specimens. Holotype, $\overparen{\sigma}$, (EJCB): "LAOS-NE, Hua Phan prov. $-20^{\circ} 12^{\prime} \mathrm{N}, 104^{\circ} 01^{\prime} \mathrm{E}$, PHU PHAN Mt., 1500-1900m, 17 v.-3.vi.2007, Vit. 

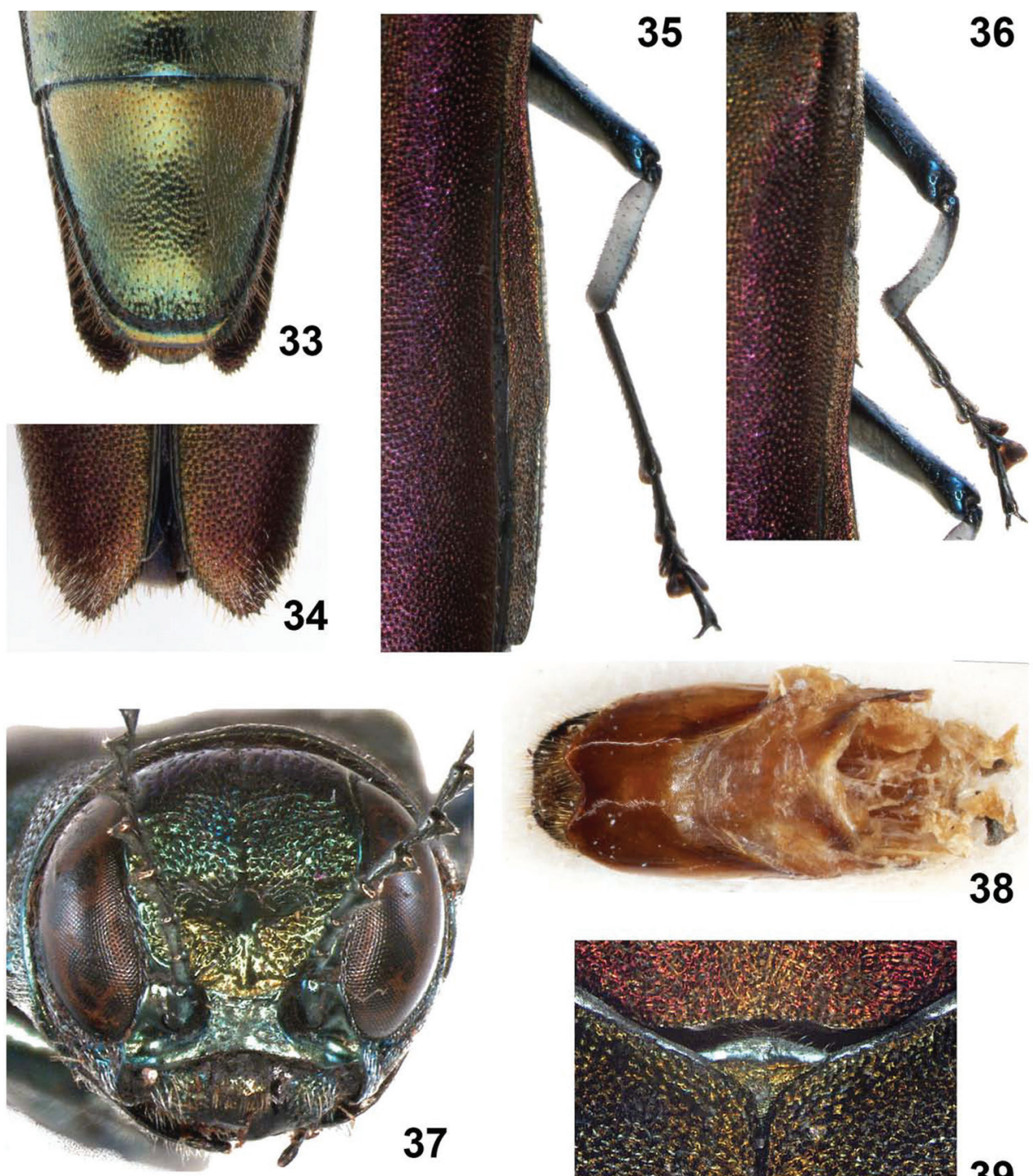

Figures 33-39. Agrilus pseudolubopetri Jendek \& Chamorro, sp. n. 33 female, last abdominal ventrite 34 female, apices of elytra 35 female, metathoracic leg 36 female, mesothoracic leg 37 male, anterior view of head 38 female, segment VIII, ventral view 39 female, scutellum.

Kubáň leg.”. Paratypes: 2 đ, 1 क , (EJCB): “LAOS-NE, Houa Phan prov., $20^{\circ} 12-$ $13.5^{\prime} \mathrm{N}, 103^{\circ} 59^{\prime} .5-104^{\circ} 01^{\prime} \mathrm{E}$, Ban Saluei $\rightarrow$ Phou Pane Mt., $1340-1870 \mathrm{~m}$, 15.iv.-

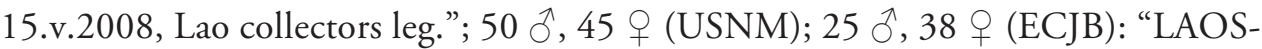
NE, Hua Phan Province, Ban Saleui, Phou Pan (Mt), $20^{\circ} 12^{\prime} \mathrm{N}, 104^{\circ} 01^{\prime} \mathrm{E}$, 7.iv25.v.2010, 1300-1900m, leg. C. Holzschuh". 42 §, 53 우 (USNM): "LAOS-NE, Hua Phan Province, Ban Saleui, Phou Pan (Mt), $20^{\circ} 12^{\prime} \mathrm{N}, 104^{\circ} 01^{\prime} \mathrm{E}, 1-31 . v .2011$, 1300-1900m, leg. C. Holzschuh". 
Distribution. Laos: Hua Phan Province.

Etymology. The specific epithet is a combination of the Greek adjective pseudos (false, lie) and-lubopetri. Name indicates relation to Agrilus lubopetri.

Agrilus sapphirinus Jendek \& Chamorro, sp. n.

urn:lsid:zoobank.org:act:76C88934-92E3-47B4-BB9D-239FEB7E839B

http://species-id.net/wiki/Agrilus_sapphirinus

Figs $40-50$

Diagnosis. This species shares several characters present in species close to Agrilus ascanius Deyrolle, 1864. Agrilus sapphirinus sp. n. can be easily differentiated by the bright metallic-blue color; lack of yellow abdominal pubescence; strongly convergent marginal and submarginal carinae; and by the dilated prosternal process with acute angles.

Description. BODY: Size: 10.5 mm (Holotype); Shape: cuneiform; Build: slender.

HEAD: Medial impression: present; Depth: moderately deep; Extent: frons; Epistoma: in plane with frons; Frons: Shape: flat; Outline: slightly protruding from head outline; Vertex: Sculpture (predominant): punctures; Aspect: semispherical; Density: sparse; Intensity: rough; Eyes: large; Shape: protruding from head outline; Lower margin: in line with antennal socket; Antennae: Length: moderate (females) Shape: slender.

PRONOTUM: Shape: transverse; sides moderately arcuate; widest subapically; anterior margin slightly narrower than posterior; Anterior lobe moderate; Shape: arcuate; Position: at level with anterior pronotal angles; Posterior angles: Apex: blunt, Shape: moderately obtuse; Disk: flat; Disk impressions: Presence: medial and lateral; Medial impression: Shape: anteromedial and posteromedial; Lateral impressions: Depth: deep; Width: wide; Prehumerus: Development: carinal; Shape: arcuate; Extent: to third of pronotal length; Anterior end: distant from pronotal angle or margin; Posterior end: distant from pronotal angle or margin; Marginal and submarginal carinae: Interspace: narrow; Convergence: strongly convergent; Junction: present; Scutellum: Size: moderate, Disk: impressed, Scutellar carina: present.

ELYTRA: Color: unicolored; Humeral carina: absent; Apices: Arrangement: separate; Shape: spinose; Position of dominant spine: medial; Elytral pubescence: absent.

STERNUM: Prosternal lobe: Size: moderate, Anterior margin: angulately emarginate, Emargination: Depth: deep; Width: wide; Prosternal process: Size: moderate; Shape: dilated; Sides: arcuate, Angles: acute, Disk: flat; Metasternal projection: flat.

ABDOMEN: Sternal groove: Shape on apex of last ventrite: arcuately sinuate; Width: narrow; Pygidium: Apical margin: arcuate.

LEGS: Metatarsus: about as long as or somewhat longer than mesotarsus; Metatarsomere1: longer than 2-4 combined.

GENITALIA: Ovipositor elongate.

Type locality. North Laos, Louang Namtha environ, N 2100.3, E 101²4.6.

Type specimens. Holotype, , (EJCB): "LAOS north, 31.v.1997, Luoang Namtha env., N2100.3, E10124.6, E. Jendek \& O. Šauša leg”. 

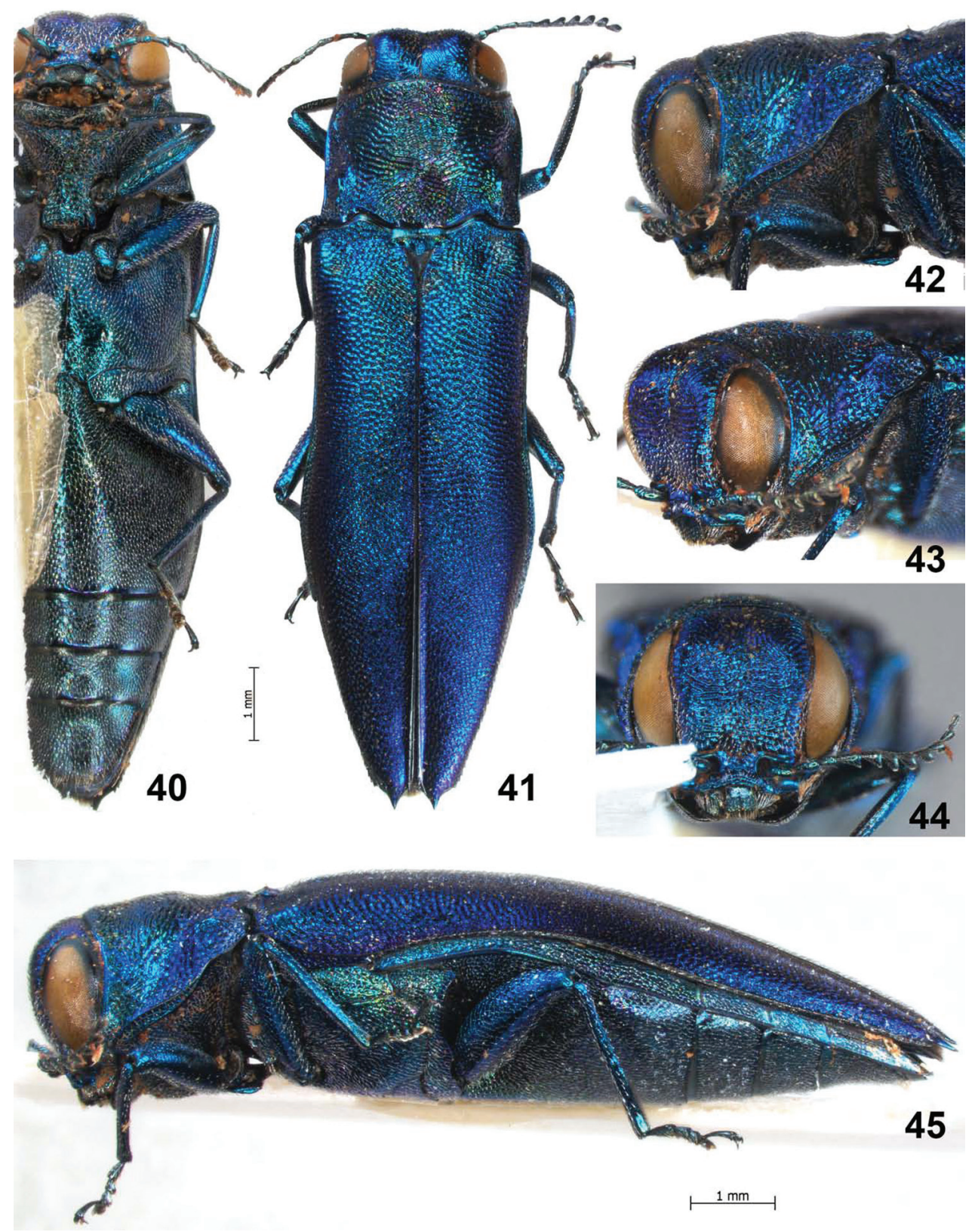

Figures 40-45. Agrilus sapphirinus Jendek \& Chamorro, sp. n. Holotype female: $\mathbf{4 0}$ ventral view $\mathbf{4 1}$ dorsal view $\mathbf{4 2}$ lateral view of head and pronotum $\mathbf{4 3}$ oblique-lateral view of head and pronotum $\mathbf{4 4}$ anterior view of head $\mathbf{4 5}$ lateral view.

Distribution. Laos: Louang Namtha Province.

Etymology. The specific epithet is Latin sapphirinus, -a, -um (of sapphire) and refers to the color of the species. 

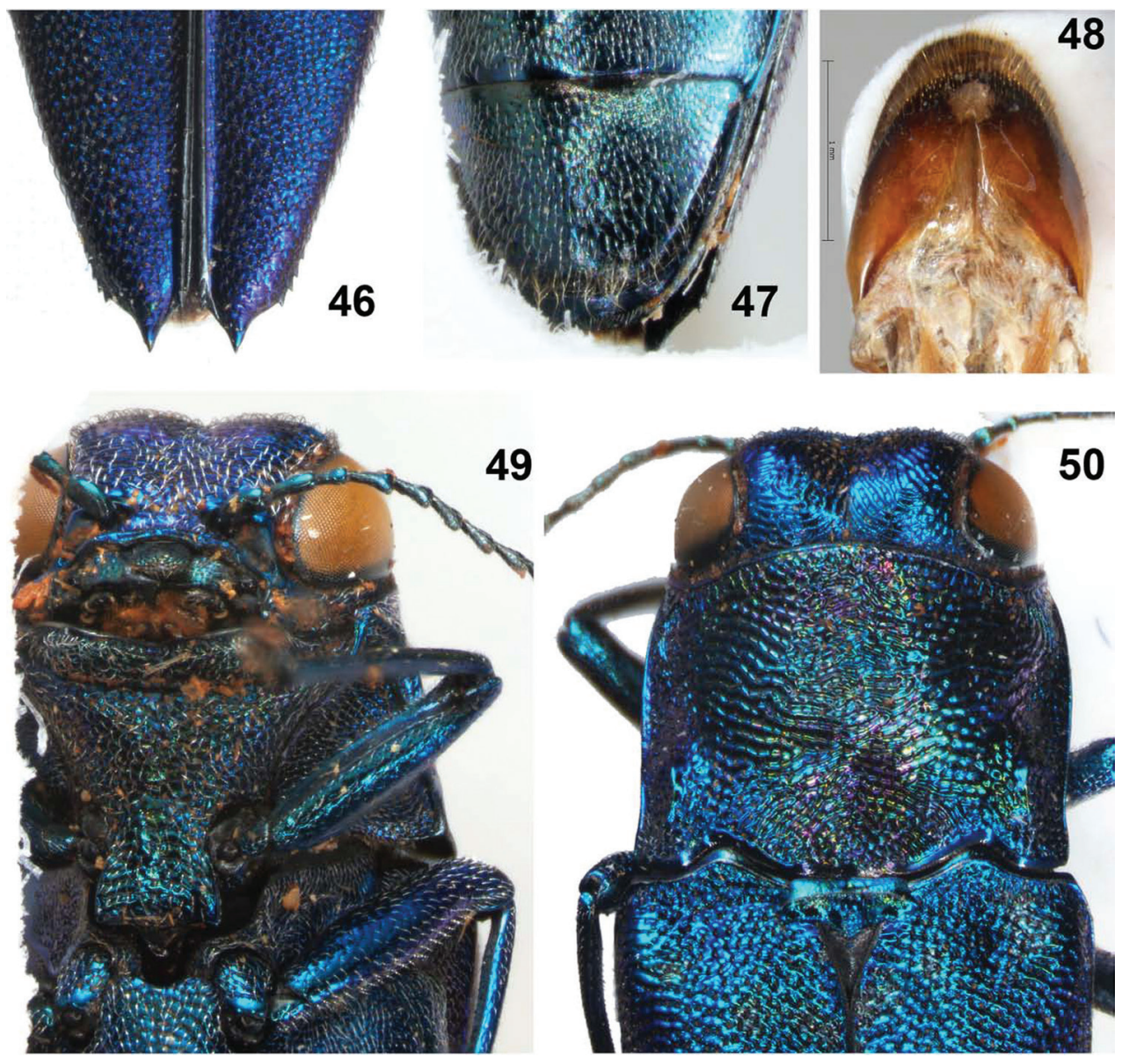

Figures 46-50. Agrilus sapphirinus Jendek \& Chamorro, sp. n. Holotype female: 46 elytral apices $\mathbf{4 7}$ last ventrite $\mathbf{4 8}$ segment VIII, ventral view $\mathbf{4 9}$ ventral view of head and prosternum $\mathbf{5 0}$ dorsal view of head, pronotum, and scutellum.

\section{Agrilus seramensis Jendek \& Chamorro, sp. $\mathbf{n}$.}

urn:Isid:zoobank.org:act:4086FAB7-63AA-47C7-BA46-2F29F9EACB23

http://species-id.net/wiki/Agrilus_seramensis

Figs $51-60$

Diagnosis. This species resembles $A$. ascanius in having the elytra markedly tapering apically; the elytral apices spinose; the pronotum almost square, disk impressions and prehumeral, marginal and submarginal carinae almost identical; a large rectangular scutellum and pronounced triangular scutellar projection; the thorax and abdomen with golden yellow tomentose patterns; and the head obviously large, metallic in color, and eyes markedly protruding. Agrilus seramensis can be differentiated by the following characters: the pronotum is green (red in A. ascanius), the elytra are green-yellow basally turning blue apically; the scutellum posterior to scutellar carina and scutel- 


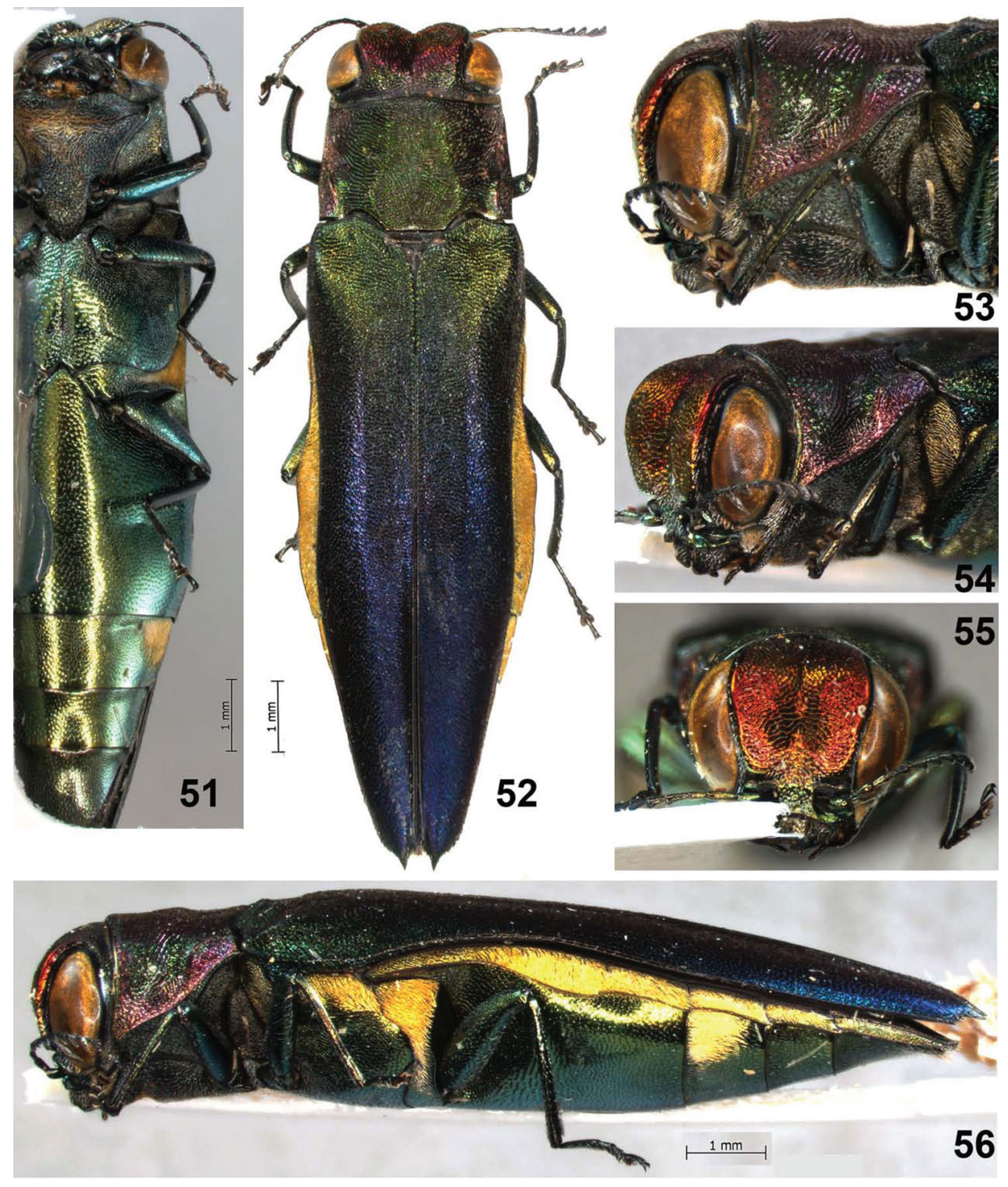

Figures $\mathbf{5}$ I-56. Agrilus seramensis Jendek \& Chamorro, sp. n. Holotype female: $\mathbf{5}$ I ventral view; 52 dorsal view $\mathbf{5 3}$ lateral view head and pronotum $\mathbf{5 4}$ oblique-lateral view of head and pronotum $\mathbf{5 5}$ anterior view of head $\mathbf{5 6}$ lateral view.

lar projection depressed; the entire scutellum black; ventrite 2 with lateral tomentose golden-yellow spots; the pronotal lateral margin straight (arcuate in $A$. ascanius); and a broader prosternal process.

Description. BODY: Size: 8.0-11.5 mm (Holotype $11 \mathrm{~mm}$ ); Shape: cuneiform; Build: slender. 

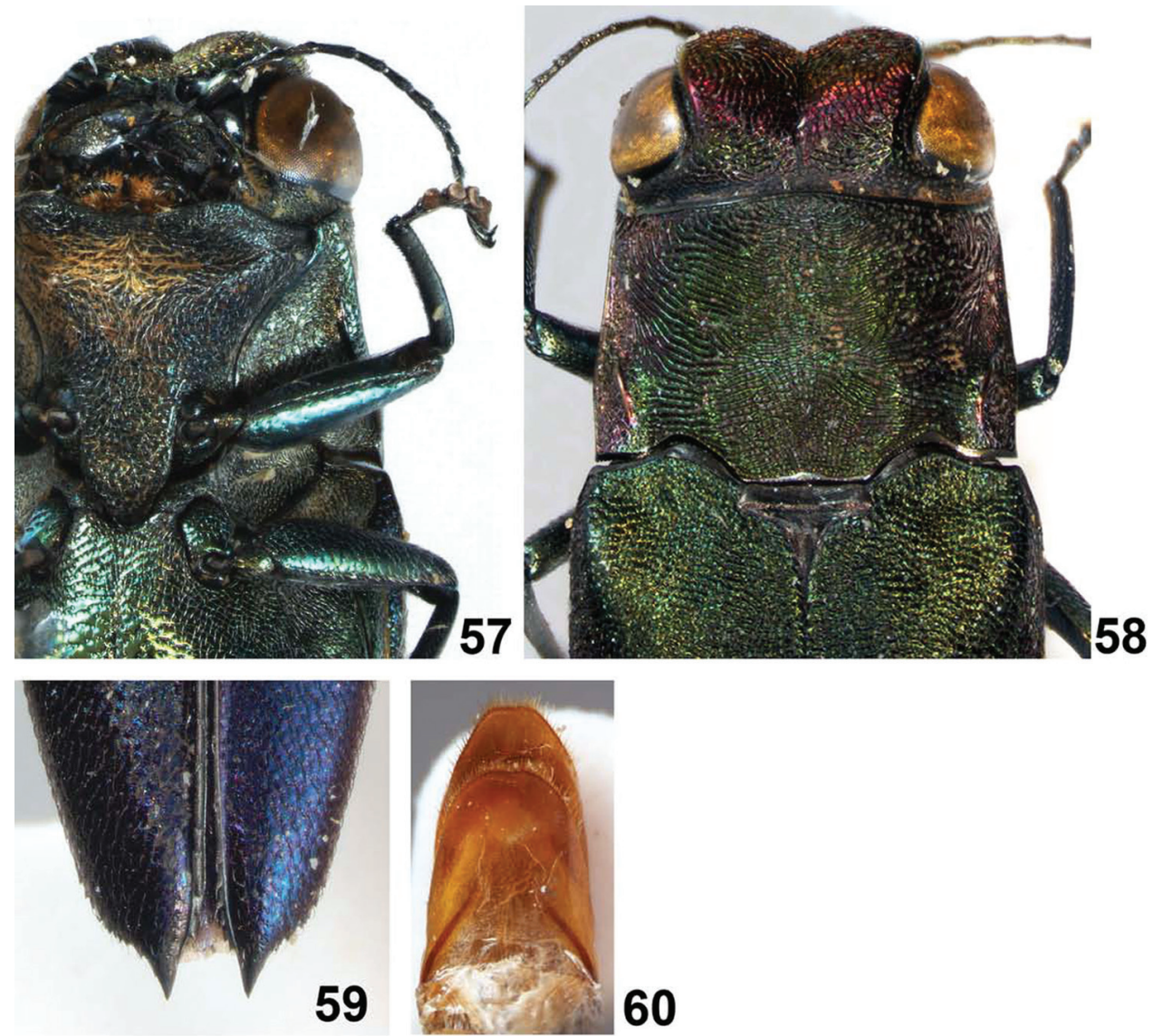

Figures 57-60. Agrilus seramensis Jendek \& Chamorro, sp. n. Holotype female: $\mathbf{5 7}$ ventral view of head and prosternum $\mathbf{5 8}$ dorsal view of head, pronotum, and scutellum 59 elytral apices $\mathbf{6 0}$ sternum VIII, ventral view.

HEAD: Shape: obviously flat; Medial impression: Depth: deep; Extent: vertex and frons; Epistoma: in plane with frons; Frons: Outline: protruding from head outline; Vertex: Outline: not protruding from head outline; Sculpture: punctures; Aspect: semispherical, Density: sparse, Intensity: rough; Eyes: Size: large; Shape: protruding from head outline; Lower margin: in line with antennal socket; Antennae: Length: moderate (females); Shape: slender.

PRONOTUM: Shape: transverse; Sides: straight; Anterior margin: narrower than posterior; Anterior lobe: moderate; Shape: arcuate; Position: at level with anterior pronotal angles; Posterior angles: Apex: sharp, Shape: acute or rectangular; Disk: Convexity: flat; Disk impressions: Presence: medial and lateral; Medial impression: Shape: anteromedial and posteromedial; Lateral impressions: Depth: deep; Width: wide; Prehumerus: Development: carinal; Extent: to third of pronotal length; Posterior end: distant from pronotal angle or margin; Anterior end: distant 
from pronotal marginal carina; Marginal and submarginal carinae: Interspace: narrow; Convergence: moderate; Junction: absent; Scutellum: Size: moderate, Disk: impressed, Carina: present.

ELYTRA: Color: bicolored; Humeral carina: absent; Apices: Arrangement: separate; Shape: spinose; Position of dominant spine: medial; Elytral pubescence: absent.

STERNUM: Prosternal lobe: Size: moderate; Anterior margin: angulately emarginate; Emargination: depth: deep; Width: wide; Prosternal process: Size: moderate; Shape: narrowed; Sides: straight; Angles: obtuse; Disk: flat.

ABDOMEN: Sternal groove: Shape on apex of last ventrite: arcuate; Pygidium: Apical margin: arcuate.

LEGS: Metatarsus: somewhat longer than mesotarsus; Metatarsomere 1: subequal to or longer than $2-4$. combined.

GENITALIA: Ovipositor: elongate.

Type locality. Indonesia, Maluku, Seram Island, $35 \mathrm{~km}$ East of Pasahari, Unit O.

Type specimens. Holotype + , (EJCB): "[Indonesia], Maluku, Seram, $35 \mathrm{~km}$ E Pasahari, Unit O, 24-30.10.1998, J. Horák leg." Paratypes: 1 q, (EJCB): “[Indonesia], Maluku, Seram, Solea, 12 km SE Wahai, 17.i-6.2.1997, S. Bílý leg”. 3 ( $\mathrm{EJCB}):$ "Indonesia], Maluku, Seram, Solea, $12 \mathrm{~km} \mathrm{SE}$ Wahai, 16.x-4.xi.1998, S. Bílý leg". 2 + (EJCB) "[Indonesia], Maluku, Seram, Solea, 12 km SE Wahai, 31.10-4.11.1998, J. Horák leg”.

Distribution. Indonesia: Maluku, Seram Island.

Etymology. The specific name is latinized adjective from the geographical term "Seram", the type locality of this species.

\section{Agrilus spineus Jendek \& Chamorro, sp. n.}

urn:Isid:zoobank.org:act:95DE2DC1-B948-4FD0-B863-CB527B92B97C

http://species-id.net/wiki/Agrilus_spineus

Figs 61-71

Diagnosis. This species is similar to Agrilus piliventris Deyrolle, 1864 in the transverse shape of the pronotum; the ventral and pleural abdominal regions completely covered by golden-yellow pubescence; the scutellum subrectangular with prominent carina; the scutellar disk and carina impressed; the scutellar projection enlarged; and the elytral apices spinose. Agrilus spineus can be distinguished from A. piliventris and by the metallic black pronotum, greenish-black elytra with minute golden dorsal pubescence; and the elytral apical spines turned medially.

Description. BODY: Size $9 \mathrm{~mm}$ (Holotype); Shape: cuneiform; Build: robust.

HEAD: Medial impression: present, Extent: frons; Epistoma: with raised upper margin; Frons: Outline: not protruding from head outline; Vertex: Outline: not protruding from head outline; Sculpture: punctures; Aspect: semispherical; Density: sparse; Intensity: rough; Eyes: Size: large; Shape: protruding from head outline; Lower margin: below antennal socket; Antennae: Length: short (female); Shape: slender. 

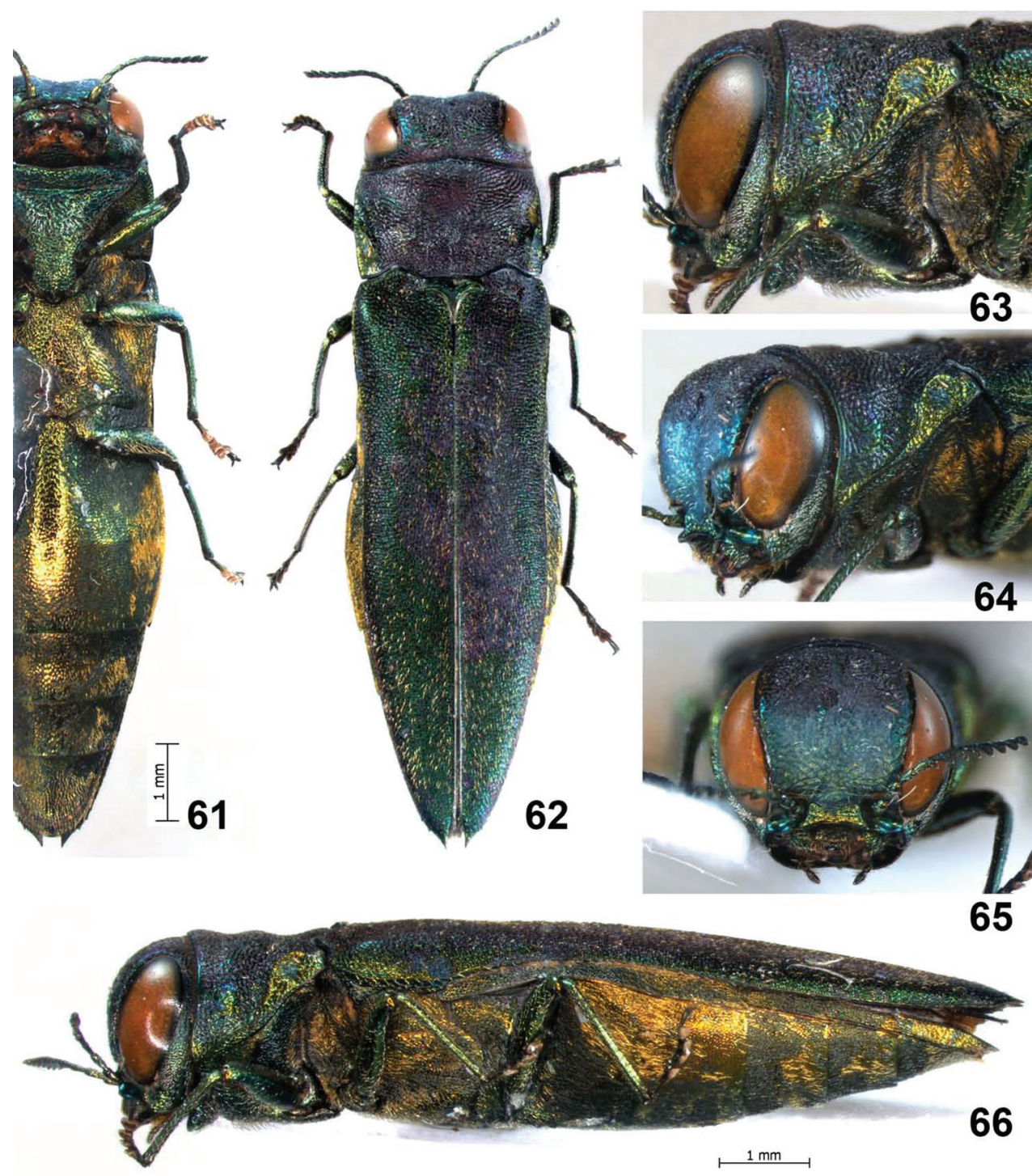

Figures 61-66. Agrilus spineus Jendek \& Chamorro, sp. n. Holotype female: $\mathbf{6 I}$ ventral view $\mathbf{6 2}$ dorsal view 63 lateral view head and pronotum 64 oblique-lateral view of head and pronotum 65 anterior view of head 66 lateral view.

PRONOTUM: Shape: transverse; Sides: arcuate; Anterior margin: narrower than posterior; Anterior lobe: moderate; Shape: arcuate; Position: at level with anterior pronotal angles; Posterior angles: Apex: blunt, Shape: obtuse; Disk: flat; Disk impressions: Presence: medial and lateral; Medial impression: Shape: anteromedial and posteromedial; Lateral impressions: Width: narrow; depth: deep; Prehumerus: Development: carinal; Shape: arcuate; Extent: to third of pronotal length; Anterior end: joining with pronotal marginal carina, Posterior end: distant from pronotal an- 


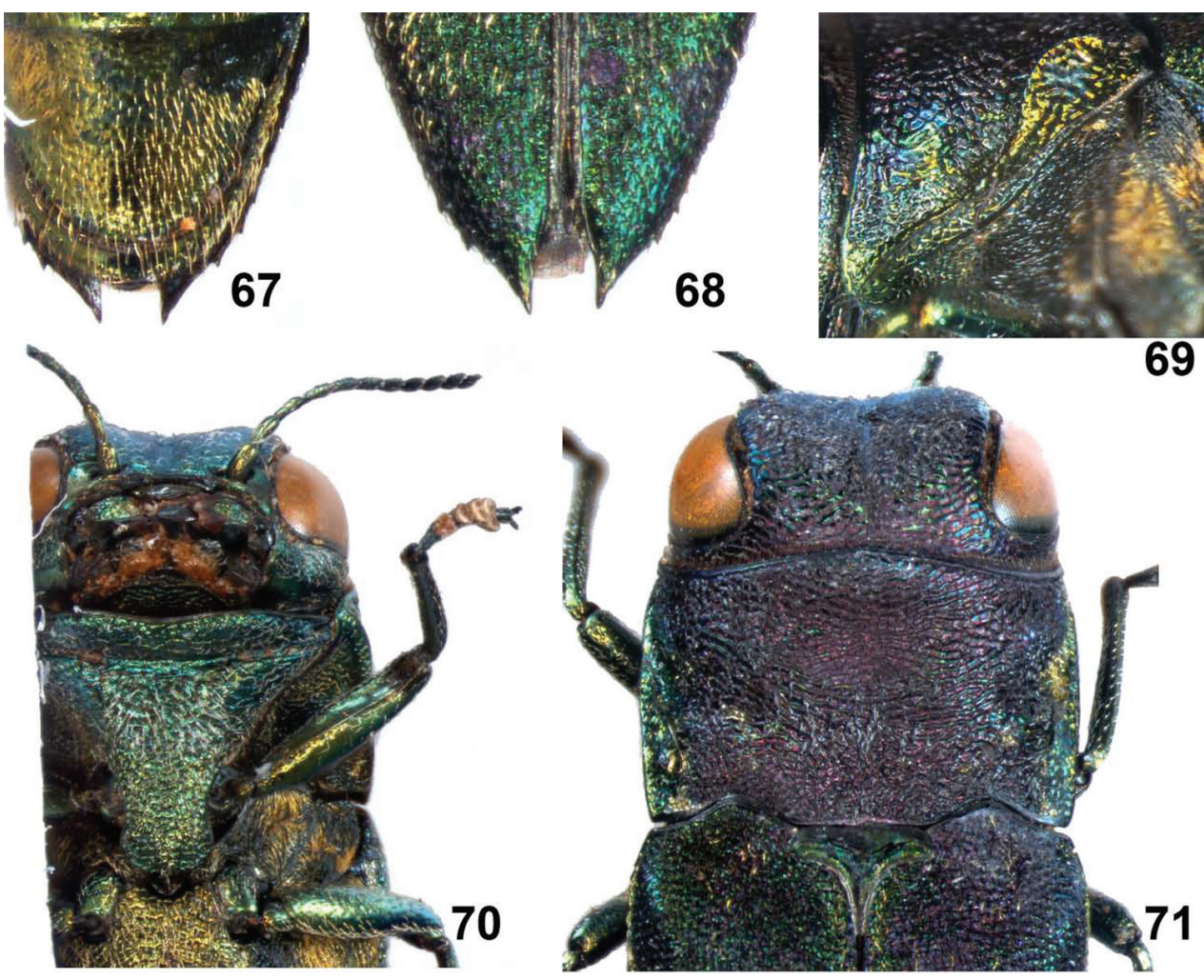

Figures 67-71. Agrilus spineus Jendek \& Chamorro, sp. n. Holotype female: 67 last ventrite 68 elytral apices $\mathbf{6 9}$ pronotal marginal, submarginal, and prehumeral carinae, oblique-lateral view $\mathbf{7 0}$ ventral view of head and prosternum $\mathbf{7 I}$ dorsal view of head, pronotum, and scutellum.

gle or margin; Marginal and submarginal carinae: Interspace: narrow; Convergence: strongly convergent; Junction: present; Scutellum: Size: moderate; Disk: impressed; Marginal carina: present or obsolete.

ELYTRA: Color: unicolored; Humeral carina: absent; Apices: Arrangement: separate; Width: narrow; Shape: spinose; Position of dominant cusp or spine: medial; Elytral pubescence: entire.

STERNUM: Prosternal lobe: Size: moderate; Anterior margin: angulately emarginate; Emargination: Depth: deep; Width: wide; Prosternal process: Size: moderate; Shape: subparallel; Sides: straight; Angles: obtuse; Disk: flat; Metasternal projection: flat.

ABDOMEN: Sternal groove: Shape on apex of last ventrite: arcuate; Pygidium: Apical margin: arcuate.

LEGS: Metatarsus: about as long as mesotarsus; Metatarsomere 1: subequal to or longer than 2-4 combined.

GENITALIA: Ovipositor: elongate.

Type locality. Malaysia, Borneo Island, Sarawak State, Bako National Park.

Type specimens. Holotype, + , (EJCB): "Borneo, Sarawak, Bako NP, 5.5.2000, M. Vyklický lgt.”. 
Distribution. Malaysia: Sarawak state

Etymology. The specific name spineus is the Latin adjective spineus, -a, -um (thorny). This refers to the spines on the elytral apices.

Agrilus tomentipennis Jendek \& Chamorro, sp. n. urn:lsid:zoobank.org:act:775E9A40-700D-4D3B-ABF4-7DF32DF066E0 http://species-id.net/wiki/Agrilus_tomentipennis Figs $72-80$

Diagnosis. Agrilus tomentipennis from Laos is very similar to A. planipennis. Both species have a small scutellum; identical marginal and submarginal carinae; a pygidial spine; and highly sinuate posterior margin of the metatibiae. While many of the differences between these two species may be considered mostly continuous, such as larger size (A. tomentipennis), larger and more pronounced prehumeral carina (A. tomentipennis); and deeper frontal concavity (A. tomentipennis), two additional features set A. tomentipennis apart from $A$. planipennis: the presence of a row of perisutural stripes of white elytral pubescence with indication of a preapical tomentose spot, and a more rectangular scutellum in $A$. tomentipennis (diamond-shaped in $A$. planipennis).

Description. BODY: Size: 14.0-14.3 mm (Holotype $14.3 \mathrm{~mm}$ ); Shape: cuneiform; Build: robust.

HEAD: Shape: flat; Medial impression: present, Depth: deep; Extent: vertex and frons; Epistoma: with raised upper margin; Frons: Shape: flat; Outline: not protruding from head outline; Vertex: Outline: not protruding from head outline; Sculpture: Predominant: punctures; Aspect: semispherical, Density: sparse, Intensity: rough; Eyes: Size: large, Shape: protruding from head outline; Lower margin: below or in line with antennal socket; Antennae: Length: moderate (females); Shape: slender.

PRONOTUM: Shape: visually square to transverse; Sides: arcuate; Maximal width: at middle; Anterior margin: narrower than posterior; Anterior lobe: moderate; Shape: arcuate; Position: at level with anterior pronotal angles; Posterior angles: Apex: blunt, Shape: obtuse; Disk: Convexity: flat, without obvious tomentose spots; Disk impressions: Presence: medial and lateral; Medial impression (shape): anteromedial and posteromedial; Lateral impression: Depth: deep; Width: narrow; Prehumerus: Development: carinal; Shape: arcuate; Extent: to third of pronotal length; Anterior end: distant from pronotal angle or margin, Posterior end: joining posterior pronotal margin; Marginal and submarginal carinae: Interspace: narrow; Convergence: strong; Junction: present; Scutellum: Size: rudimentary, Disk: not impressed, Scutellar carina: obsolete.

ELYTRA: Color: unicolored; Humeral carina: absent; Apices: Arrangement: separate; Shape: arcuate; Elytral pubescence: perisutural stripes; Color: unicolored; Character: with spots of denser pubescence. STERNUM: Prosternal lobe: moderate; Anterior margin: arcuately emarginate; Depth: deep; Width: wide. Prosternal process: Size: moderatee; Shape: narrowed or subparallel; Sides: straight; Angles: obtuse; Disk: flat; Metasternal projection: flat. 

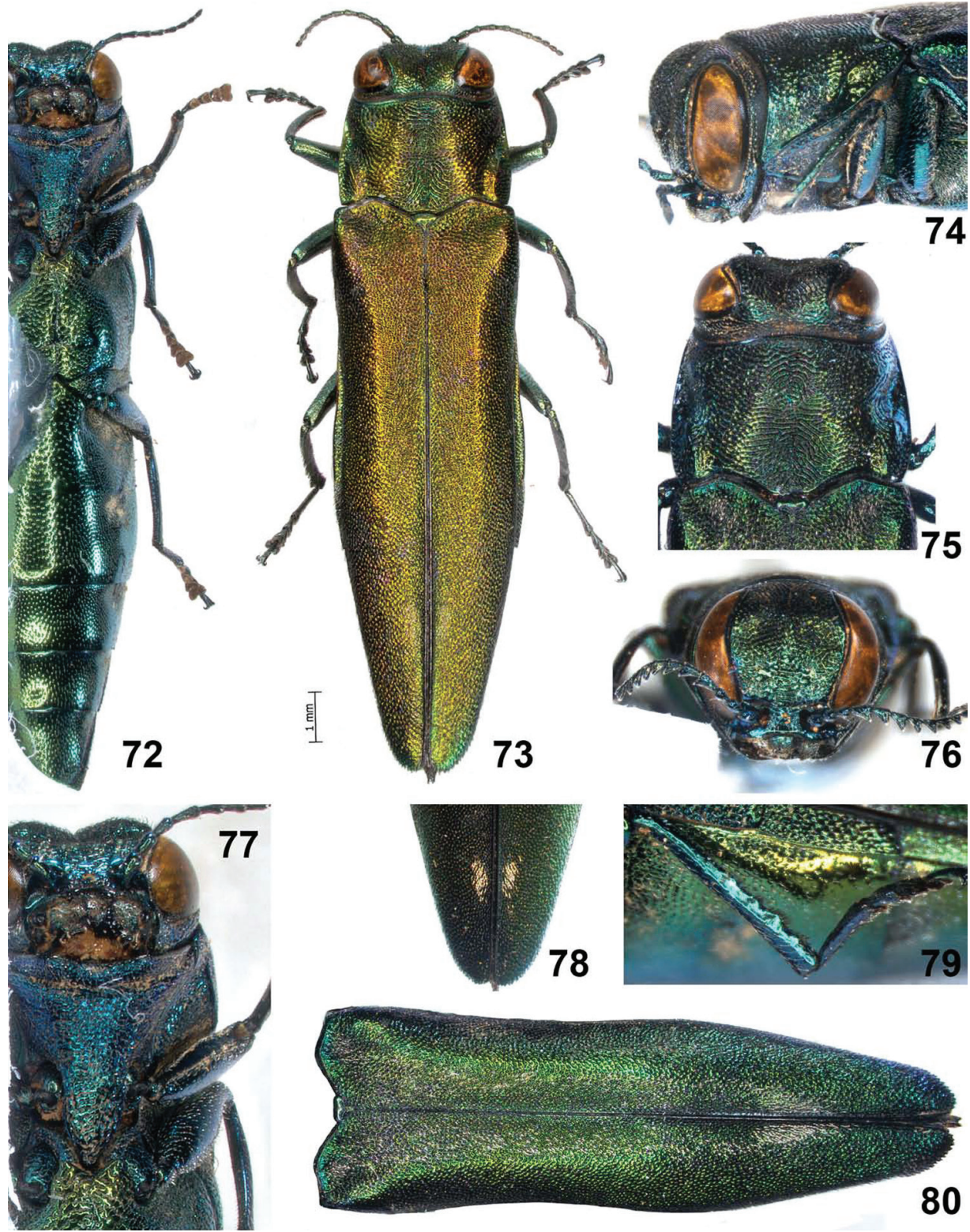

Figures 72-80. Agrilus tomentipennis Jendek \& Chamorro, sp. n. Holotype female: $\mathbf{7 2}$ ventral view $\mathbf{7 3}$ dorsal view $\mathbf{7 4}$ lateral view head and pronotum $\mathbf{7 5}$ dorsal view of head, pronotum, and scutellum $\mathbf{7 6}$ anterior view of head $\mathbf{7 7}$ ventral view of head prosternum $\mathbf{7 8}$ detail of apex of elytra showing tomentose spots best visible at angle, dorsal view $\mathbf{7 9}$ metathoracic leg with sinuate posterior margin $\mathbf{8 0}$ elytra, dorsal view (female paratype).

ABDOMEN: Sternal groove: Shape on apex of last ventrite: arcuate, Pygidium: extended into long spine.

LEGS: Metatarsus: distinctly longer than mesotarsus; Metatarsomere 1: subequal to or longer than 2-4 combined. 
GENITALIA: Ovipositor: elongate.

Type locality. Northeastern Laos, Xieng Khouang province, $45 \mathrm{~km}$ Eastern of Phonsavan: Ban Namseung.

Type specimens. Holotype + , (EJCB): "LAOS-NE, Xieng Khouang prov., 45 km (by road) E of Phonsavan: $-1000 \mathrm{~m}$ Ban Namseung, April 2008, Ch. Keomaravong leg". Paratypes: 1 +, (USNM): "LAOS-NE, Xieng Khouang prov., 45 km (by road) E. of Phonsavan: $1000 \mathrm{~m}$ Ban Namseung, April 2008, Ch. Keomaravong leg”. 1 , (NMPC): "LAOS-NE, Xieng Khouang prov., $45 \mathrm{~km}$ (by road) E. of Phonsavan: $-1000 \mathrm{~m}$ Ban Namseung, vi.2011, Ch. Keomaravong leg”.

Distribution. Laos: Xieng Khouang Province.

Etymology. The specific epithet is a combination of Latin nouns tomentum (woolly hairs) and pennae (elytra). The name alludes to the presence of white pubescence on the elytra.

Remarks. Jendek and Grebennikov (2011) cited specimens of this taxon in examined material as $A$. planipennis.

\section{Acknowledgments}

For consultation, providing specimens, and/or hosting our visits, we thank Aleš Smetana \& Vasily Grebennikov (Ottawa Plant Laboratory, Canadian Food Inspection Agency); Norman Woodley, Steve Lingafelter, Alexander Konstantinov, Allen Norrbom, \& Alma Solís (Systematic Entomology Laboratory, Agriculture Research Service, USDA); Antoine Mantilleri \& Isabelle Bruneau de Miré (Muséum national d'Histoire naturelle, Paris); Malcolm Kerley, Sharon Shute and Max Barclay (The Natural History Museum, London); and Vit Kubáň and Jiř́i Hájek (National Museum of Natural History, Prague). Finally, we thank Kevin Hackett (National Programs Staff, ARS), Dana Roth and Darcy Nelson (Forest Service, International Programs) for their efforts to secure funding for this project. USDA is an equal opportunity provider and employer.

\section{References}

Baudon A (1963) Addenda à la liste des Buprestidae du Laos. Bulletin de la Société Royale des Sciences Naturelles du Laos 9: 49-74.

Baudon A (1965) Contribution a l'etude des Buprestidae du Laos (Coleoptera) (1 re partie). Bulletin \& Annales de la Societe Royale Belge d'Entomologie 101: 193-230.

Baudon A (1968) Catalogue commenté des Buprestidae récoltés au Laos. Deuxième Partie. Vientiane, Ministère de l'Information, 190 pp.

Bellamy CL (2003) An Illustrated Summary of the Higher Classification of the Superfamily Buprestoidea (Coleoptera). Folia Heyrovskyana Supplementum 10: 1-197.

Bellamy CL (2008) A world catalogue and bibliography of the jewel beetles (Coleoptera: Buprestoidea). Volume 4, Agrilinae: Agrilina through Trachyini. Pensoft, Sofia, 1932-2684. 
Curtis J (1825) British Entomology; Being illustrations and Descriptions of the Genera of Insects found in Great Britain and Ireland: containing Coloured Figures from Nature of the most Rare and Beautiful Species, and in many instances of the Plants upon which they are found. Printed for the Author by Richard and John E. Taylor, London, 98 color pls with text.

Descarpentries A, Villiers A (1967) Catalogue raisonné des Buprestidae d'Indochine. XII. Agrilini (supplément). Bulletin du Muséum National d'Histoire Naturelle, Paris, 2e Série, 39 (1): $143-150$.

Deyrolle H (1864) Description des Buprestides de la Malaisie recueillés par M. Wallace pendant son voyage dans cet Archipel. Annales de la Société entomologique de Belgique 8: 1-280.

Fairmaire L (1888) Notes sur les Coléoptères des environs de Pékin. Revue D’Entomologie 7: $111-160$

Jendek E (2000) Revision of the Agrilus cyaneoniger species group. Entomological Problems 31: $187-197$.

Jendek E (2006) Taxonomic and nomenclatural notes on the genera Sarawakita Obenberger and Agrilus Curtis (Coleoptera: Buprestidae: Agrilinae). Zootaxa 1153: 33-42.

Jendek E, Grebennikov VV (2011) Agrilus (Coleoptera, Buprestidae) of East Asia. Jan Farkac, Prague, $362 \mathrm{pp}$.

Kerremans C (1912) A contribution to the study of the insect fauna of Borneo. A list of the Bornean Buprestidae (Part II.). Sarawak Museum Journal 1: 68-82.

Kubáň V, Majer K, Kolibáč J (2000) Classification of the tribe Coraebini Bedel, 1921 (Coleoptera, Buprestidae, Agrilinae). Acta Musei Moraviae Scientiae Biologicae 85: 185-287.

Obenberger J (1924) Druhá řada nových rodů čeledi Buprestidae (Coleoptera). Deuxième série de nouveaux genres de Buprestides. Acta Entomologica Musaei Nationalis Pragae 2: 7-44. Obenberger J (1936) Buprestidae V. In: Junk W, Schenkling S (Eds) Coleopterorum Catalogus. Verlag für Naturwissenschaften, W. Junk, Gravenhage, 935-1246.

Obenberger J (1960) Notes et études buprestologiques I. (Coleoptera). Buprestodologické studie I. Acta Universitatis Carolinae, Biologica 2: 125-153.

Ohmomo S (2002) Informations of insects from Thailand (Part 3). Otoshibumi, Tsukuba, 22, 21-29. [in Japanese with English subtitle and summary] 